\title{
Serum biomarkers of the calcium-deficient rats identified by metabolomics based on UPLC/Q-TOF MS/MS
}

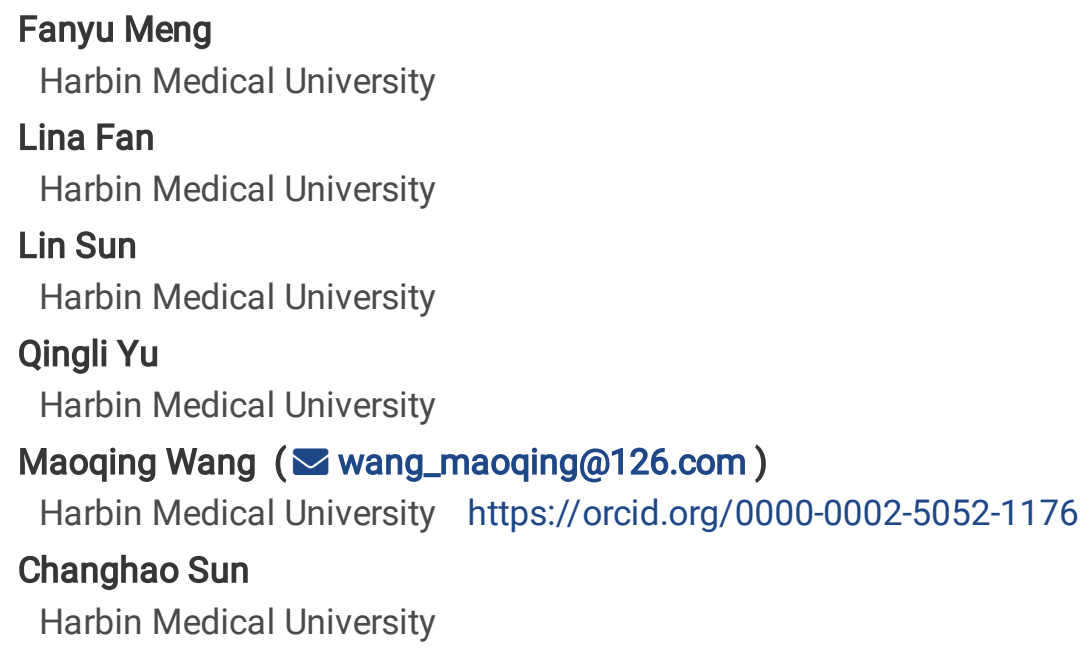

\section{Research}

Keywords: biomarkers, calcium deficiency, metabolomics, UPLC/Q-TOF MS/MS, serum

Posted Date: December 30th, 2019

DOI: https://doi.org/10.21203/rs.2.19585/v1

License: (c) (i) This work is licensed under a Creative Commons Attribution 4.0 International License. Read Full License 


\section{Abstract}

Background: We previously identified the urinary biomarkers to diagnose calcium deficiency and nutritional rickets by ultraperformance liquid chromatography/quadrupole time-of-flight tandem mass spectrometry (UPLC/Q-TOF MS/MS).To further confirm these biomarkers in vivo, we performed serum metabolomics analysis of calcium deficiency.

Methods: A calcium-deficient rat model was established with a low-calcium diet for 12 weeks. Serum-metabolomics-based UPLC/Q-TOF MS/MS and multivariate statistical analysis was performed to identify the alterations in metabolites associated with calcium deficiency in rats.

Results: Bone mineral density, serum parathyroid hormone and alkaline phosphatase were significantly decreased in the lowcalcium diet group (LCG) compared to the normal calcium diet group (NCG). Serum metabolic-profiling analysis could definitively distinguish between the LCG and NCG and identified 25 calcium-deficient biomarkers. Three metabolites (indoxyl sulfate, phosphate, and taurine) of the 25 biomarkers were found in our previous urinary metabolomics study of rats with a calcium deficiency and nutritional rickets. The areas under the curve (AUCs) of these three biomarkers were greater than 0.8, and the combination of any two biomarkers was higher than 0.95 .

Conclusion: Dietary calcium deficiency induced the alterations of metabolites in the serum of rats, and the three identified biomarkers had relatively high diagnostic values for calcium deficiency in rats.

\section{Introduction}

Calcium is a major constituent of bones and teeth and also plays an essential role as a second messenger in cell-signaling pathways $^{[1]}$. An adequate intake of calcium is indispensable for human health. Calcium deficiency is a worldwide nutritionaldeficiency public-health problem ${ }^{[2]}$. Similar to other countries, calcium deficiency is common in China ${ }^{[3,4]}$. Over the past 20 years, the calcium intake of Chinese adults has not been sufficient, with a large gap between the actual intake and recommended intake of calcium. The proportions of citizens who satisfy the recommended appropriate intake (RNI) have been reported to be less than $7 \%$ in adult males, less than $5 \%$ in adult females and less than $4 \%$ in older adults over the age of $50^{[5,6]}$. Long-term calcium deficiency affects the health of the body and decreases the bone mineral density (BMD), with adverse outcomes such as rickets ${ }^{[7,8]}$ and osteoporosis ${ }^{[9-11]}$. Early or slight calcium deficiency has no obvious clinical signs or symptoms and can be prevented. Therefore, early diagnosis and discovery of calcium deficiency is necessary for therapy.

Current methods for assessing calcium nutrition status mainly include dietary survey, calcium balance measurement ${ }^{[12,13]}$, serum biochemical tests(calcium, alkaline phosphatase (AP), and parathyroid hormone (PTH)) and BMD. However, the above methods have their limitations and cannot be widely used in population research, especially for slight calcium deficiency ${ }^{[14,15]}$. The calcium balance test takes at least three days, which is time consuming and laborious and is therefore not suitable for screening people with calcium deficiency. The body has a strong ability to maintain calcium homeostasis in the body. Blood calcium is relatively constant and is normal in the early stage of calcium deficiency. Decreased BMD is the result of long-term calcium deficiency. When serum calcium and BMD change significantly, the body's calcium deficiency is already severe, causing some irreversible pathological changes to the body. Therefore, it is necessary to find new calciumdeficient biomarkers and establish a rapid, sensitive and specific method for evaluation of calcium nutritional status.

Metabolomics can detect all metabolites in biological samples through high-throughput detection techniques, uncover subtle metabolic changes in the body caused by pathophysiology or external stimuli, and clearly reveal the relationship between metabolite changes and the physiological and pathological state of the body ${ }^{[16-19]}$. Metabolomics methods based on ultrahigh performance liquid chromatography/mass spectrometry (UPLC/MS) have the advantages of high sensitivity, high dynamic range, etc., and have been widely used for the screening of various disease biomarkers, such as calcium deficiency ${ }^{[20]}$, rickets ${ }^{[21]}$, postprandial hyperglycemia ${ }^{[22]}$, diabetes ${ }^{[23,24]}$,Alzheimer's disease ${ }^{[25]}$, autism ${ }^{[26]}$ and cancer ${ }^{[27]}$. 
In our previous urinary metabolomics study of calcium-deficient rats, an integrated metabolomics strategy for identifying reliable biomarkers of calcium deficiency was established. A total of 27 reliable calcium-deficient biomarkers were identified, two of which were verified in menopausal females ${ }^{[22]}$. In our urinary metabolomics study of nutritional rickets, 31 biomarkers of nutritional rickets were identified, and five candidate biomarkers for clinical diagnosis were screened by receiver operating characteristics (ROC)analysis ${ }^{[28]}$. In the validation step, the urinary levels of these five candidate biomarkers indicated that the combination of phosphate and sebacic acid provided accurate diagnostic capability with high sensitivity and specificity. In additionally, 15 biomarkers in urine of calcium-deficient rats were repeated in the urines of infants with rickets. The results verified that calcium deficiency is an important cause of rickets. So far, however, there has been no metabolomics analysis of calcium-deficient serum.

In this study, we used the same metabolomics method as before to analyze the changed metabolites in the serum of calcium-deficient rats, to reveal calcium-deficient biomarkers, and to identify reliable biomarkers compared with the biomarkers from the urine metabolomics study. We expect to build an accurate assessment method of calcium nutritional status based on these biomarkers in the future.

\section{Methods}

\section{Animal experiments}

Twenty male Wistar rats (five weeks old, 90-110 g) were purchased from Beijing Vital River Laboratory Animal Technology Co., Ltd, Beijing, China. All rats were housed in single cages. The temperature of the animal room was kept at $22 \pm 1{ }^{\circ} \mathrm{C}$, the relative humidity was $60 \%$, the illumination period was $12 \mathrm{~h}$, and light and dark periods were alternated. After 7 days of adaptive feeding, the 20 rats were randomly divided into two groups. The normal calcium diet group (NCG, 10rats) was fed with a normal calcium diet $(0.50 \%(\mathrm{w} / \mathrm{w})$ calcium), and the low-calcium diet group (LCG)was fed with a low-calcium diet $(0.15 \%(\mathrm{w} / \mathrm{w})$ calcium)for 12 weeks. The animal diets were modified based on the standard AIN-93Gdiet from Beijing KeAoLiXie Animal Food Co.,Ltd., Beijing, China. The rats were given free access to water, and their food intake was recorded regularly. At the end of the experiment, all rats were anesthetized by intraperitoneal injection of sodium pentobarbital (40 mg/kg body weight)(Tianjin Guangfu Fine Chemical Research Institute) after fasting for 12 hours and were then sacrificed by exsanguination. Blood samples from the abdominal aorta was collected and centrifuged at $3000 \mathrm{rpm}$ ( $835 \mathrm{~g}$ ) for $15 \mathrm{~min}$. The serum was separated and stored in a refrigerator at $-80^{\circ} \mathrm{C}$. The serum calcium and phosphorus levels of all rats were measured by anautomatic biochemical analyzer (Hitachi 7100 Automatic Biochemistry Analyzer (Hitachi High Technologies, International Trading, Shanghai, China), and PTH levels was detected by an Elisa kit(SUMMUS, Harbin). The left femur of each rat was isolated by dissection. The femur BMDs of rats were measured using dual energy X-ray absorptiometry (Norland XR-36DEXA System; Cooper Surgical, Trumball, CT, USA) in the Second Affiliated Hospital of Harbin Medical University. The study was approved by the Harbin Medical University Institutional Animal Care Committee and performed in accordance with the Harbin Medical University guidelines for the care and use of laboratory animals.

\section{Serum metabolomics by UPLC/Q-TOF MS/MS Pretreatment of serum samples}

All serum samples were thawed at room temperature and then vortexed for $1 \mathrm{~min}$. Then, $700 \mu \mathrm{L}$ methanol (HPLC, Thermo Fisher) was added to $350 \mathrm{Ml}$ serum to precipitate protein. After vortexing for $1 \mathrm{~min}$, all serum samples were centrifuged at12000 r/min for $10 \mathrm{~min}$, and the supernatants were transferred into $2 \mathrm{~mL}$ tube. After drying with nitrogen, the residue in the $2 \mathrm{ml}$ tube was dissolved by $350 \mu \mathrm{L}$ of a mixed solution of water : acetonitrile (2:1). After centrifugation at $12000 \mathrm{r} / \mathrm{min} \mathrm{for}$ $10 \mathrm{~min}$, the supernatants were transferred to a sample bottle for testing.

\section{UPLC analysis conditions}


Chromatographic separation was performed on a $1.8 \mu \mathrm{m}$ BEH C18 column (ACQUITY (HSS); Waters Corp., Milford, MA, USA; $2.1 \mathrm{~mm} \times 100 \mathrm{~mm}$ ) used with an ACQUITY UPLC system (Waters Corp., Milford, MA, USA). The temperatures of the column and auto-sampler were maintained at $35^{\circ} \mathrm{C}$ and $4{ }^{\circ} \mathrm{C}$, respectively.

Mobile phase A was ultrapure water (containing $0.1 \%$ formic acid, Tianjin Comeio); mobile phase B was chromatographically pure acetonitrile (HPLC, Thermo Fisher); and the flow rate was $0.35 \mathrm{~mL} / \mathrm{min}$. The metabolites in serum were separated by using the gradient elution method in positive and negative ion mode (Sup. Table 1). Each injection was 2 $\mu \mathrm{L}$, the column temperature was $35^{\circ} \mathrm{C}$, and the autosampler temperature was $4{ }^{\circ} \mathrm{C}$.Acetonitrile was run every fifth sample as a blank solution, and the serum samples were injected alternately as five NCG and five LCG samples.

\section{Mass spectrometry conditions}

Q-TOF MS/MS was performed with Micromass Q-TOF mass spectrometer(Waters Corp., Manchester, UK) using an electrospray ionization (ESI) interface. The MS data were collected in Centroid mode both in positive and negative ion mode. The analytical parameters of Q-TOF mass spectrometry were as follows: capillary voltage $3.0 \mathrm{kV}\left(\mathrm{ESI}^{+}\right) / 2.8 \mathrm{kV}\left(\mathrm{ESI}^{-}\right)$; $\mathrm{cone}^{\circ}$ voltage $35 \mathrm{~V}$; extraction cone voltage $3 \mathrm{~V}$; ion source temperature $125^{\circ} \mathrm{C}$; desolvation gas temperature $320^{\circ} \mathrm{C}$; desolvation gas $\left(\mathrm{N}_{2}\right)$ flow rate,700L/hr; cone gas (nitrogen) flow, $50 \mathrm{~L} / \mathrm{h}$; collision gas, argon; MCP detector voltage, $2350 \mathrm{~V}$; collision energy, $6 \mathrm{~V}$; scanning time, $0.4 \mathrm{~s}$; and scanning time interval, $0.1 \mathrm{~s}$. To ensure the accuracy and repeatability of the mass-to-charge ratio, a concentration of $200 \mathrm{pg} / \mathrm{ml}$ leucine-cerebral peptide solution (Waters) was used as the Lock-Spray calibration solution, and the exact mass-to-charge ratios in positive and negative ion modes were respectively $[\mathrm{M}+\mathrm{H}]^{+}=556.2771$ and $[\mathrm{M}+\mathrm{H}]^{-}=554.2615$. The lock spray frequency was set at 10 seconds, and the lock mass data were averaged over 10 scans for correction. The data acquisition range was $\mathrm{m} / \mathrm{z} 50 \sim 1000$, and the acquisition time was $0 \sim 16 \mathrm{~min}$.

\section{Data analysis}

After the data acquisition by the liquid chromatography-mass spectrometer was completed, MakerLynx software (incorporated into the MassLynx software; version 4.1; SCN 714; Waters Corp., USA)was used for peak matching, alignment, and other pretreatment. TheApexTrack peak parameters were set as follows: peak width at $5 \%$ height, $1 \mathrm{~s}$; and peak-to-peak baseline noise as calculated automatically. The collection parameters were set as follows: mass window, $0.05 \mathrm{Da}$; retention time window, 0.2 min; minimum intensity, 80; noise elimination level, 6.0; deisotope data, "Yes". Data were used for the period of 0.4 to $10.5 \mathrm{~min}$. After recognition and alignment, the intensity of each ion was normalized to the summed total ion intensity of each chromatogram to take into account variations in urine concentration and volume. Multivariate statistical analysis such as principal component analysis (PCA) and partial least-squares discriminant analysis (PLS-DA) was performed using EZ-info software (version 2.0.0.0; June 5, 2008; Waters Corp., USA) to obtain the classification trends of the two groups. SIMCA-P software (version 11.5; Umetrics AB, Umeå, Sweden) was used in across-validation procedure with 200 random permutations for avoiding the overfitting of supervised PLS-DA models. Biomarkers were screened by orthogonal partial least squares (OPLS-DA).

\section{Identification of biomarkers}

The accurate mass and MSMS spectrum of the markers were determined by quadrupole/time-of-flight mass spectrometry. Compound databases such as the Human Metabolome Database (HMDB) (http://www.hmdb.ca) and Metlin (http://metlin.scripps.edu/) and ChemSpider (http://www.chemspider.com)were searched by molecular formula or molecular mass to obtain possible compound structures. The chemical structures of the markers were identified fromstandard compounds based on both retention times and MS/MS spectra.

\section{Statistical analysis}

The serum biochemical indicators and BMD of two groups are expressed as $x \pm S D$. The statistical difference between the two groups was analyzed by an independent sample t test. A two-tailed threshold of $p<0.05$ was considered statistically different between the two groups. The ROC analysis and other statistical analysis were implemented using the R language. 
All analyses including the error bars of three unique metabolites were performed using SPSS software (version 16.0; SPSS Inc., Chicago, IL, USA).

\section{Results}

\section{Biochemical analysis and BMD}

Compared with the normal calcium diet group (NCG), significant differences for serum PTH, AP and BMD were observed in the low-calcium diet group (LCG) (Table 1). There were no significant differences between the two groups in terms of the serum calcium or body weight.

\section{Metabolic profiling analysis}

The serum samples of all rats were analyzed by ultra-performance liquid chromatography/quadrupole time-of-flight tandem mass spectrometry (UPLC/Q-TOF MS/MS) in the positive $\left(\mathrm{ESI}^{+}\right)$and negative ion ( $\left.\mathrm{ESI}^{-}\right)$modes. To visualize the general clustering trends between the two groups, principal component analysis (PCA) was applied to the serum metabolic profile. As shown in Figure $1 A$ and B, the PCA score plots revealed clear clustering trends between the LCG and NCG for metabolites in both the positive ion mode (for the first two components:R2Y=27.3\%, Q2=5.0\%) and the negative ion mode(for the firstcomponent:R2Y=34.4\%, Q2=13.4\%). Partial least-squares discriminant analysis (PLS-DA) score plots showed significant classification trends between the two groups (Figure $1 \mathrm{C}$ ) in ESI ${ }^{+}$(Figure 1C,one principal component,R2Y=99.4\%, Q2=86.5\%) and $\mathrm{ESI}^{-}$(Figure 1D, one principal component, R2Y=99.5\%, Q2=92.6\%).

As shown in Figure 2, all permuted Q2s were below or at approximately 0 for the positive and negative modes, and all R2Ys and Q2s were much lower than the original points to the right. The validation plots generated from 200 permutation tests supported the validity of the PLS-DA mode for both $\mathrm{ESI}^{+}$and $\mathrm{ESI}^{-}$(Figure 2). Our results indicated that calcium deficiency induced distinct metabolite alterations in the serum of rats.

Based on the VIP values of orthogonal partial least-squares discriminant analysis (OPLS-DA) models (VIP $<1.5)$ and $p$ values of independent-sample t-tests ( $p<0.05), 59$ differential variables in $\mathrm{ESI}^{+}$and 71 in $\mathrm{ESI}^{-}$(Supp Table 2-3) were screened in serum. Among them, 25 metabolites ( 9 downregulated and 16 upregulated in calcium-deficient rats) were confirmed by available databases and standard compounds (Table 2). The heatmap visualization (Figure 3) of 25 biomarkers between the two groups showed distinct segregation.

ROC analysis was used to evaluate the predictive ability of all potential metabolic biomarkers (Supp Table 2-3). The ROC range of the 25 markers of difference was 0.722 to 0.956 , indicating that the screened material had strong diagnostic ability for calcium-deficient rats. We screened three biomarkers, namely, indoxyl sulfate(area under the curve (AUC) $=0.951$, sensitivity $=0.889$, specificity $=0.900)$, phosphoric acid $(A U C=0.800$, sensitivity $=1.000$, specificity $=0.500)$ and taurine $(A U C=0.889$, sensitivity $=0.889$, specificity $=0.900$ ), with AUC values of 0.8 or greater (Figure 4).

\section{Discussion}

Calcium deficiency is a worldwide nutritional deficiency problem. However, the current diagnosis methods of calcium deficiency still have some shortcomings, so these methods cannot be widely used in population research of early-stage calcium deficiency. Therefore, there is an urgent need to find new calcium deficiency biomarkers for establishing a new accurate diagnostic method. Our previous metabolomics experiments found potential diagnostic biomarkers in urine for calcium deficiency in calcium deficient rat and infant nutritional rickets. Although urine biomarkers are conveniently and noninvasively obtained compared to blood biomarkers, urine metabolites may be affected by diet and diseases and may be not as stabilized as in serum. If the biomarkers found in the urine also appear in the blood, these biomarkers may be suitable 
for future clinical studies. To evaluate whether these biomarkers from urine appear in serum, we performed serum metabolomics analysis of calcium-deficient rats.

As shown by our study results, 25 identified metabolites were chosen as reliable biomarkers of calcium deficiency in serum. Among shown in Fig. 5, three biomarkers, including indoxyl sulfate, phosphoric acid and taurine, also appeared in the urine of both calcium-deficient rats and infants with nutritional rickets. Thus, these three biomarkers were considered reliable biomarkers, independent of specimen and species changes. We can detect the levels of these three biomarkers in the urine or blood and can meet the evaluation requirements of the body's calcium-deficiency status.

Indoxyl sulfate and phosphoric acid increased in both urine and serum when the rats were calcium deficient. Furthermore, taurine decreased in urine yet increased in serum.

In earlier studies, it was found that indoxyl sulfate can disrupt the function of osteoblasts and induce abnormal bone exchange ${ }^{[29,30]}$. A low-calcium diet may disturb the function of osteoblasts and normal bone exchange, and the BMD of the LCG was indeed reduced. Our results suggest that osteoblast function and bone turnover of the LCG rats could be impaired by increased indoxyl sulfate. Phosphorus is involved in biological processes of bone mineralization. Calcium and phosphorus are normally in balance ${ }^{[31]}$. Low calcium-to-phosphorus ratios have been found to increase parathyroid hormone (PTH) secretion and urinary calcium excretion, and decrease BMD. In our previous study of calcium deficiency, increased pyrophosphate, CAMP, and phosphate in the urine of the LCG rats indicated that low calcium diet impaired calcium and phosphorus metabolism ${ }^{[20]}$. Increased PTH inhibited the re-absorption of phosphorus and increased urinary phosphate excretion. In this study, increased serum phosphoric acid was observed in LZG compared with NZG. Our results found that increased phosphoric acid in both serum and urine of LCG was associated with decreased BMD. Mi-Ja Choi etal. Has reported that taurine facilitates the transport of calcium ions and influences bone metabolism ${ }^{[32-34]}$. In our study, the increased taurine in the serum of the LCG indicated that the body needs high concentrations of taurine to maintain the body's calcium level and bone density and that the excretion of taurine was reduced. Therefore, decreased taurine in the urine of calcium-deficient rats was observed. In summary, indophenol sulfate, phosphate, and taurine were closely related to the decreased BMD by calcium deficiency. In addition to the above three metabolites, six pathways (valine, leucine and isoleucine biosynthesis; biosynthesis of unsaturated fatty acids; valine, leucine and isoleucine degradation; aminoacyl-tRNA biosynthesis; pyruvate metabolism; and phenylalanine, tyrosine and tryptophan biosynthesis) were enriched by pathway analysis (Fig. 6 and Sup Table 4).

The ROC results indicated that these three biomarkers (i.e., indoxyl sulfate, phosphate, and taurine) can facilitate the diagnosis of calcium-deficient rats (AUC > 0.8, Fig. 4) and could provide a diagnosis index of calcium deficiency. To further improve the diagnostic ability, a comprehensive diagnostic analysis of the three markers was performed. Logistic regression was used to combine several different variables into a multivariable parameter. When the three markers were combined, the AUC of two or three markers was greater than 0.95 , and the diagnostic ability was significantly improved compared with that of a single AUC $(p<0.05)$. In addition, there was no statistical difference in AUC values between the two combinations of the two markers $(p>0.05)$, indicating that in these three differential markers, the combination of any two biomarkers has high diagnostic value. Therefore, we can select the appropriate combination of the three markers for the diagnosis of calcium deficiency according to the actual situation to aid in diagnosing calcium deficiency in people.

\section{Conclusion}

In this study, we carried out an unbiased global serum metabolomics analysis on calcium-deficient rats and identified 25 reliable biomarkers. Indoxyl sulfate, phosphate, and taurine were previously found in the urine of calcium-deficient rats and infants with rickets and have the potential to be used in the diagnosis of calcium deficiency in the population.

\section{Abbreviations}


AP: alkaline phosphatase; PTH: parathyroid hormone; BMD: bone-mineral density; LCG: low-calcium group; NCG: normalcalcium group UPLC/Q-TOF MSMS:ultra-performance liquid chromatography/quadrupole time-of-flight tandem mass spectrometry

\section{Declarations}

\section{Acknowledgements}

The authors thank AiMi Academic Services (www.aimieditor.com) for English language editing and review services.

\section{Authors' contributions}

Maoqing Wang and Changhao Sun contributed to the study's conception and design. Fanyu Meng and Lina Fan contributed to drafting of the manuscript. Lin Sun and QingliYu, performed animal experiments and data collection. Fanyu Meng and Lina Fan performed data analysis and interpretation. The final draft was read and approved by all the authors.

\section{Funding}

This work was supported by grants from National Nature Science Foundation of China (81573147).

\section{Availability of data and materials}

The datasets used and/or analyzed during the current study are available from the corresponding author on reasonable request.

\section{Ethics approval}

This animal study was performed according to institutional guidelines with the approval of the Institutional Animal Care and Use Committee of the Harbin Medical University.

\section{Consent for publication}

Not applicable.

\section{Competing interests}

All authors have declared that no conflict of interest exists.

\section{References}

[1] Heaney RP, Weaver CM. Newer perspectives on calcium nutrition and bone quality. Journal of the American College of Nutrition. 2005;24:574S-81S. 
[2] Ma J, Johns RA, Stafford RS. Americans are not meeting current calcium recommendations. The American journal of clinical nutrition. 2007;85:1361-6.

[3] Yuna HE, Zhai F, Wang Z, Yisong HU. Status of dietary calcium intake of Chinese residents. Wei sheng yan jiu = Journal of hygiene research. 2007;36:600.

[4] Zhai FY, He YN, Wang ZH, Yu WT, Yang XG. The status and trends of dietary nutrients intake of Chinese population.

[5] Zhang J, Wang HJ, Wang ZH, Zhang JG, Du WW, Su C, et al. [Trend in dietary calcium intake among Chinese children and adolescents aged 4 to 17 years in nine provinces from 1991 to 2009]. 2013;34:857-62.

[6] Zhang J, Wang HJ, Wang ZH, Zhang JG, Du WW, Su C, et al. [Trend in dietary calcium intake among Chinese elderly aged 50 years and over in nine provinces, from 1991 to 2009]. Zhonghua liu xing bing xue za zhi = Zhonghua liuxingbingxue zazhi. 2012;33:1119-22.

[7] Marie PJ, Pettifor JM, Ross FP, Glorieux FH. Histological osteomalacia due to dietary calcium deficiency in children. The New England journal of medicine. 1982;307:584-8.

[8] Thacher TD. Calcium-Deficiency Rickets. 2003;6:105.

[9] Tai V, Leung W, Grey A, Reid IR, Bolland MJ. Calcium intake and bone mineral density: systematic review and metaanalysis. Bmj. 2015;351:h4183.

[10] Press OU. CALCIUM DEFICIENCY IN THE ETIOLOGY OF OSTEOPOROSIS. Nutrition Reviews. 2009;21:181-3.

[11] Burckhardt P. [Calcium in the prevention and treatment of osteoporosis]. 2010;231:169-80.

[12] Hunt CD, Johnson LAK. Calcium requirements: new estimations for men and women by cross-sectional statistical analyses of calcium balance data from metabolic studies. 2007;86:1054-63.

[13] Hunt, Curtiss D, Johnson, Luann K. Calcium requirement: new estimations for men and women by cross-sectional analyses of metabolic calcium balance data. 2007;21:A173-A4.

[14] Strand MA, Perry J, Jin M, Tracer DP, Fischer PR, Zhang P, et al. Diagnosis of rickets and reassessment of prevalence among rural children in northern China. Pediatrics international : official journal of the Japan Pediatric Society. 2007;49:2029.

[15] Iba K, Takada J, Yamashita T. The serum level of bone-specific alkaline phosphatase activity is associated with aortic calcification in osteoporosis patients. Journal of bone and mineral metabolism. 2004;22:594-6.

[16] Yang J, Sun X, Feng Z, Hao D, Wang M, Zhao X, et al. Metabolomic analysis of the toxic effects of chronic exposure to low-level dichlorvos on rats using ultra-performance liquid chromatography-mass spectrometry. Toxicology letters. 2011;206:306-13.

[17] Wu R, Wu Z, Wang X, Yang P, Yu D, Zhao C, et al. Metabolomic analysis reveals that carnitines are key regulatory metabolites in phase transition of the locusts. Proceedings of the National Academy of Sciences of the United States of America. 2012;109:3259-63.

[18] Nicholson JK, Lindon JC, Holmes E. \"Metabonomics\": understanding the metabolic responses of living systems to pathophysiological stimuli via multivariate statistical analysis of biological NMR spectroscopic data. Xenobiotica.29:1181-9.

[19] Wilson ID, Gika H, Theodoridis G, Plumb RS, Shockcor J, Holmes E, et al. Global metabolic profiling procedures for urine using UPLC-MS.5:1005-18. 
[20] Wang M, Yang X, Wang F, Li R, Ning H, Na L, et al. Calcium-deficiency assessment and biomarker identification by an integrated urinary metabonomics analysis. BMC medicine. 2013;11:86.

[21] Wang M, Yang X, Ren L, Li S, He X, Wu X, et al. Biomarkers identified by urinary metabonomics for noninvasive diagnosis of nutritional rickets. Journal of proteome research. 2014;13:4131-42.

[22] Liu L, Wang M, Yang X, Bi M, Na L, Niu Y, et al. Fasting serum lipid and dehydroepiandrosterone sulfate as important metabolites for detecting isolated postchallenge diabetes: serum metabolomics via ultra-high-performance LC-MS. Clinical chemistry. 2013;59:1338-48.

[23] Claudino WM, Quattrone A, Biganzoli L, Pestrin M, Bertini I, Di Leo A. Metabolomics: Available Results, Current Research Projects in Breast Cancer, and Future Applications. Journal of Clinical Oncology.25:2840-6.

[24] Roberts LD, Koulman A, Griffin JL. Towards metabolic biomarkers of insulin resistance and type 2 diabetes: progress from the metabolome. Lancet Diabetes \& Endocrinology.2:65-75.

[25] Cui Y, Liu X, Wang M, Liu L, Sun X, Ma L, et al. Lysophosphatidylcholine and Amide as Metabolites for Detecting Alzheimer Disease Using Ultrahigh-Performance Liquid Chromatography-Quadrupole Time-of-Flight Mass SpectrometryBased Metabonomics. Journal of Neuropathology \& Experimental Neurology.73:954-63.

[26] Wang H, Liang S, Wang M, Gao J, Wu L. Potential serum biomarkers from a metabolomics study of autism. Journal of Psychiatry \& Neuroscience Jpn. 2015;40:140009.

[27] Hart CD, Vignoli A, Tenori L, Uy GL, Leo AD. Serum Metabolomic Profiles Identify ER-Positive Early Breast Cancer Patients at Increased Risk of Disease Recurrence in a Multicenter Population. Clinical Cancer Research An Official Journal of the American Association for Cancer Research. 2017;23.

[28] Wang M, Yang X, Ren L, Li S, He X, Wu X, et al. Biomarkers Identified by Urinary Metabonomics for Noninvasive Diagnosis of Nutritional Rickets. Journal of proteome research.13:4131-42.

[29] Nii-Kono T, Iwasaki Y, Uchida M, Fujieda A, Hosokawa A, Motojima M, et al. Indoxyl sulfate induces skeletal resistance to parathyroid hormone in cultured osteoblastic cells. Kidney international. 2007;71:738-43.

[30] Muteliefu G, Enomoto A, Jiang P, Takahashi M, Niwa T. Indoxyl sulphate induces oxidative stress and the expression of osteoblast-specific proteins in vascular smooth muscle cells. Nephrol Dial Transplant.24:2051-8.

[31] Kemi VE, Karkkainen MU, Rita HJ, Laaksonen MM, Outila TA, Lamberg-Allardt CJ. Low calcium:phosphorus ratio in habitual diets affects serum parathyroid hormone concentration and calcium metabolism in healthy women with adequate calcium intake. The British journal of nutrition. 2010;103:561-8.

[32] Choi MJ. Taurine May Modulate Bone in Cholesterol Fed Estrogen Deficiency-Induced Rats. Advances in experimental medicine and biology. 2017;975 Pt 2:1093-102.

[33] Yuan LQ, Xie H, Luo XH, Wu XP, Zhou HD, Lu Y, et al. Taurine transporter is expressed in osteoblasts. Amino acids. 2006;31:157-63.

[34] Yuan LQ, Lu Y, Luo X-H, Xie H, Wu X-P, Liao E-Y. Taurine promotes connective tissue growth factor (CTGF) expression in osteoblasts through the ERK signal pathway. Amino acids.32:425-30.

\section{Tables}

Table 1 Body weight, serum biological indicators and bone-mineral density between NCG and LCG after 12 
weeks.

\begin{tabular}{ccc}
\hline Characteristic & Normal-calcium group & Low-calcium group \\
\hline Body weight & $484.6 \pm 28.91$ & $468.25 \pm 41.34$ \\
Calcium, mmol/l & $2.22 \pm 0.14$ & $2.27 \pm 0.10$ \\
$\mathrm{AP}, \mathrm{U} / \mathrm{l}$ & $80.1 \pm 16.3$ & $117.8 \pm 17.9^{*}$ \\
\hline $\mathrm{PTH}, \mathrm{pg} / \mathrm{ml}$ & $31.2 \pm 1.75$ & $42.8 \pm 2.13^{*}$ \\
$\mathrm{BMD}, \mathrm{g} / \mathrm{cm}^{2}$ & $0.29 \pm 0.02$ & $0.27 \pm 0.03^{*}$ \\
\hline
\end{tabular}

Abbreviations: AP, alkaline phosphatase; PTH, parathyroid hormone; BMD, bone-mineral density; *Significant differences ( $P<0.05)$ for mean values of low-calcium group with normal-calcium group.

'able 2 The identified 25 biomarkers of calcium deficiency in serum

\begin{tabular}{|c|c|c|c|c|c|c|c|c|c|c|}
\hline $\begin{array}{l}\text { Ion } \\
\text { mode }\end{array}$ & RTĐmin $\square$ & $\begin{array}{c}\text { Actual } \\
\text { mass } \square \text { Dal }\end{array}$ & $\begin{array}{l}\text { Exact } \\
\text { mass } \\
(\mathrm{Da})\end{array}$ & $\begin{array}{l}\text { Mass } \\
\text { error } \\
(\mathrm{ppm})\end{array}$ & $\begin{array}{c}\text { Elemental } \\
\text { composition }\end{array}$ & $\begin{array}{c}\text { Metabolite } \\
\text { identification }\end{array}$ & $\begin{array}{l}\text { Change } \\
\text { trend }\end{array}$ & $\begin{array}{c}\text { Fold } \\
\text { change }\end{array}$ & $P$ & VIP \\
\hline+ & 0.72 & 204.1242 & 204.1230 & 6 & C9H17NO4 & L-Acetylcarnitine & $\downarrow$ & 0.67 & 0.000 & 4.44 \\
\hline+ & 0.76 & 258.1115 & 258.1084 & 12 & C10H15N3O5 & 5-Methylcytidine & $\uparrow$ & 1.42 & 0.001 & 3.23 \\
\hline+ & 0.77 & 118.0875 & 118.0863 & 10 & C5H11NO2 & L-Valine & $\uparrow$ & 1.35 & 0.001 & 4.44 \\
\hline+ & 0.78 & 148.0641 & 148.0624 & 11 & C6H7N5O & 7-Methylguanine & $\uparrow$ & 3.88 & 0.001 & 3.56 \\
\hline+ & 0.79 & 203.0627 & 203.0662 & 17 & C7H10N2O5 & Penmacric acid & $\downarrow$ & 0.65 & 0.000 & 3.12 \\
\hline- & 0.81 & 124.0076 & 124.0074 & 2 & C2H7NO3S & Taurine & $\uparrow$ & 1.22 & 0.004 & 1.62 \\
\hline+ & 0.94 & 262.1298 & 262.1285 & 5 & C11H19NO6 & Methylmalonylcarnitine & $\uparrow$ & 1.40 & 0.041 & 2.82 \\
\hline+ & 0.98 & 182.0822 & 182.0812 & 6 & C9H11NO3 & L-Tyrosine & $\uparrow$ & 1.14 & 0.041 & 1.91 \\
\hline+ & 1.00 & 132.1039 & 132.1019 & 15 & $\mathrm{C} 6 \mathrm{H} 13 \mathrm{NO} 2$ & L-Leucine & $\downarrow$ & 0.95 & 0.046 & 1.52 \\
\hline- & 1.21 & 115.0048 & 115.0037 & 10 & $\mathrm{C} 4 \mathrm{H} 4 \mathrm{O} 4$ & Fumaric acid & $\uparrow$ & 5.45 & 0.000 & 1.68 \\
\hline- & 1.21 & 89.0231 & 89.0244 & 15 & $\mathrm{C} 3 \mathrm{H} 6 \mathrm{O} 3$ & L-Lactic acid & $\uparrow$ & 1.21 & 0.000 & 2.93 \\
\hline- & 1.53 & 194.9450 & 194.9465 & 8 & H6P2O8 & Phosphoric acid & $\uparrow$ & 2.82 & 0.027 & 1.94 \\
\hline+ & 2.93 & 157.0630 & 157.0614 & 9 & C6H10N2O4 & N-Acetylasparagine & $\downarrow$ & 0.00 & 0.000 & 2.80 \\
\hline- & 3.39 & 241.1158 & 241.1188 & 13 & $\mathrm{C} 11 \mathrm{H} 20 \mathrm{~N} 2 \mathrm{O} 5$ & $\begin{array}{l}\text { gamma- } \\
\text { Glutamylisoleucine }\end{array}$ & $\downarrow$ & 0.01 & 0.000 & 12.28 \\
\hline- & 4.01 & 283.0701 & 283.0684 & 6 & $\mathrm{C} 16 \mathrm{H} 12 \mathrm{~N} 4 \mathrm{O} 6$ & Xanthosine & $\uparrow$ & 2.38 & 0.002 & 2.52 \\
\hline- & 4.87 & 129.0565 & 129.0557 & 6 & C6H10O3 & Ketoleucine & $\uparrow$ & 1.52 & 0.000 & 2.69 \\
\hline- & 4.87 & 281.0902 & 281.0891 & 4 & $\mathrm{C} 11 \mathrm{H} 14 \mathrm{~N} 4 \mathrm{O} 5$ & 1-Methylinosine & $\uparrow$ & 2.17 & 0.000 & 3.22 \\
\hline- & 4.96 & 225.1139 & 225.1132 & 3 & C12H18O4 & $\begin{array}{l}\text { 3,4-Methylenesebacic } \\
\text { acid }\end{array}$ & $\downarrow$ & 0.02 & 0.000 & 9.36 \\
\hline- & 4.98 & 293.1120 & 293.1143 & 8 & $\mathrm{C} 14 \mathrm{H} 18 \mathrm{~N} 2 \mathrm{O} 5$ & $\begin{array}{l}\text { gamma- } \\
\text { Glutamylphenylalanine }\end{array}$ & $\downarrow$ & 0.03 & 0.000 & 3.62 \\
\hline- & 5.59 & 212.0009 & 212.0023 & 7 & C8H7NO4S & Indoxyl sulfate & $\uparrow$ & 2.05 & 0.001 & 2.31 \\
\hline- & 6.64 & 301.2172 & 301.2173 & 0 & C20H30O2 & Eicosapentaenoic acid & $\uparrow$ & 1.50 & 0.001 & 2.85 \\
\hline+ & 7.76 & 548.3729 & 548.3711 & 3 & C28H54NO7P & LysoPC(20:2(11Z,14Z)) & $\uparrow$ & 1.63 & 0.000 & 4.36 \\
\hline- & 9.63 & 327.2293 & 327.2330 & 11 & $\mathrm{C} 22 \mathrm{H} 32 \mathrm{O} 2$ & Docosahexaenoic acid & $\uparrow$ & 1.53 & 0.001 & 6.52 \\
\hline- & 9.96 & 303.2297 & 303.2330 & 12 & $\mathrm{C} 20 \mathrm{H} 32 \mathrm{O} 2$ & Arachidonic acid & $\downarrow$ & 0.95 & 0.032 & 2.99 \\
\hline- & 10.25 & 279.2289 & 279.2330 & 15 & $\mathrm{C} 18 \mathrm{H} 32 \mathrm{O} 2$ & Linoleic acid & $\downarrow$ & 0.82 & 0.028 & 3.68 \\
\hline
\end{tabular}

Note: Abbreviations: RT, retention time (minute). Arrow denoted the increase $(\uparrow)$ or decrease $(\downarrow)$ of the metabolites in lowcalcium diet rat compared with those in the control rats

\section{Figures}


ESI+
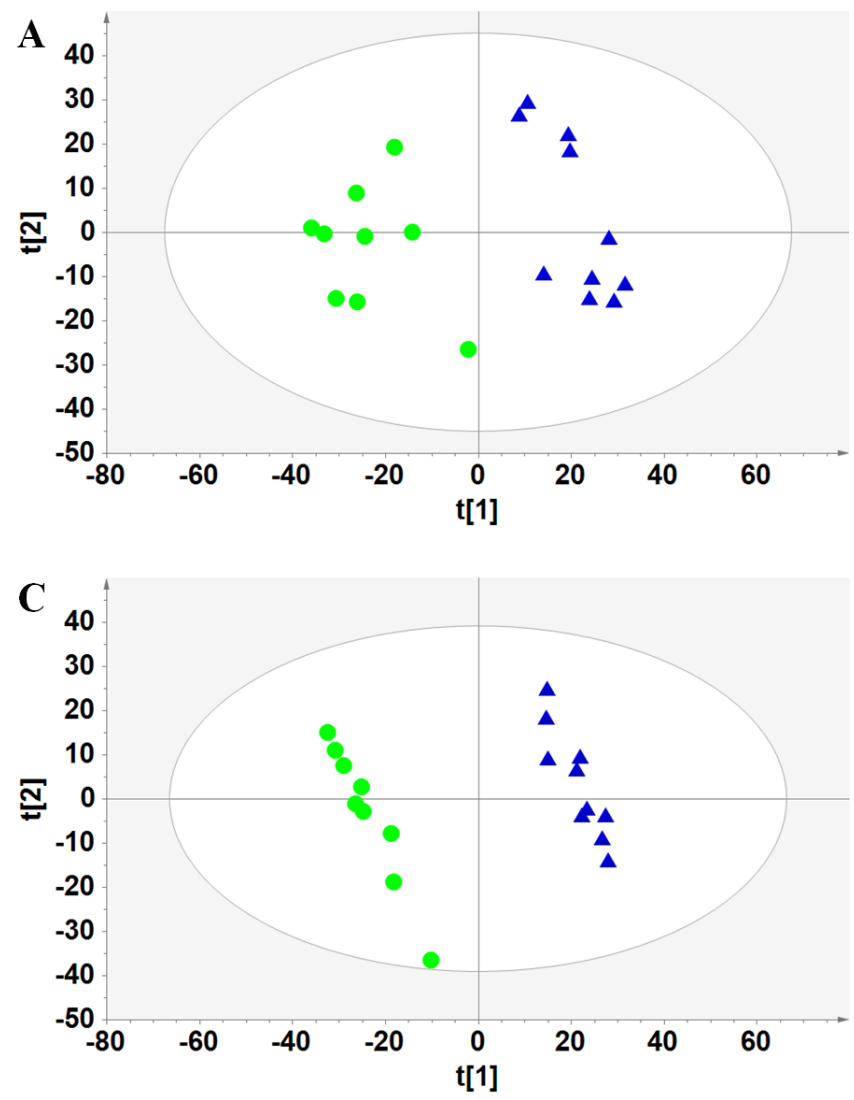

ESI-

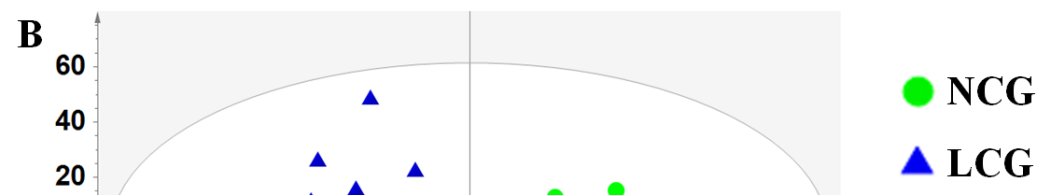

\section{Figure 1}

The PCA and PLS-DA score plots of analysis of the LCG and NCG. PCA score plot in ESI+ (A) and ESI-(B): PLS-DA score plot in ESI+ (C) and ESI- (D). Blue triangles: LCG. Green circles: NCG; each data point stands for one sample.

\section{ESI+}

A

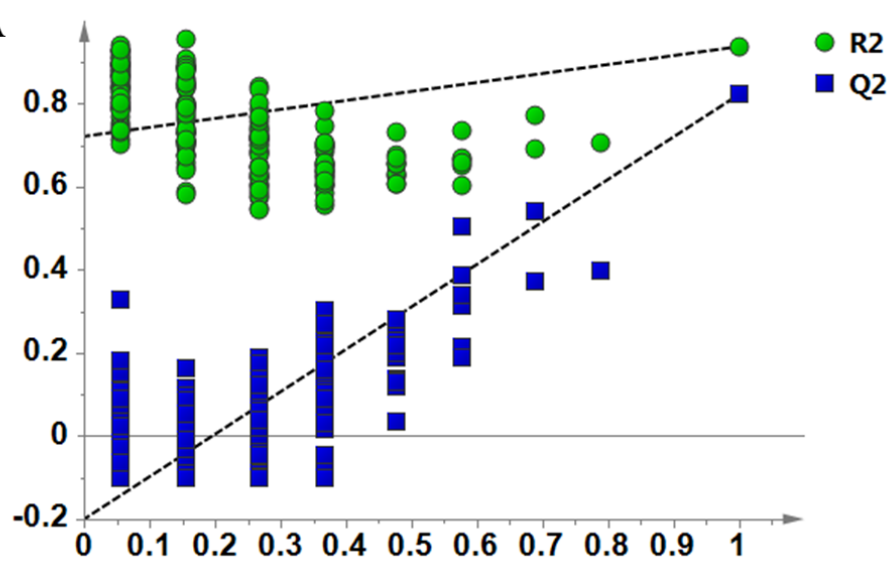

ESI-

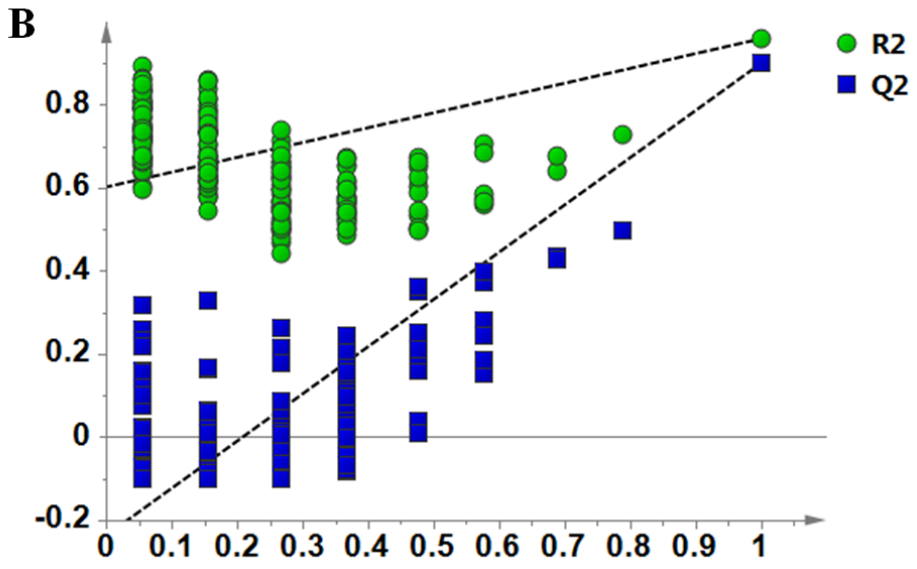

Figure 2

The permutation plots of the PLS-DA model. The R2Y value represents the goodness of fit of the model, and the Q2 value represents the predictability of the model. 


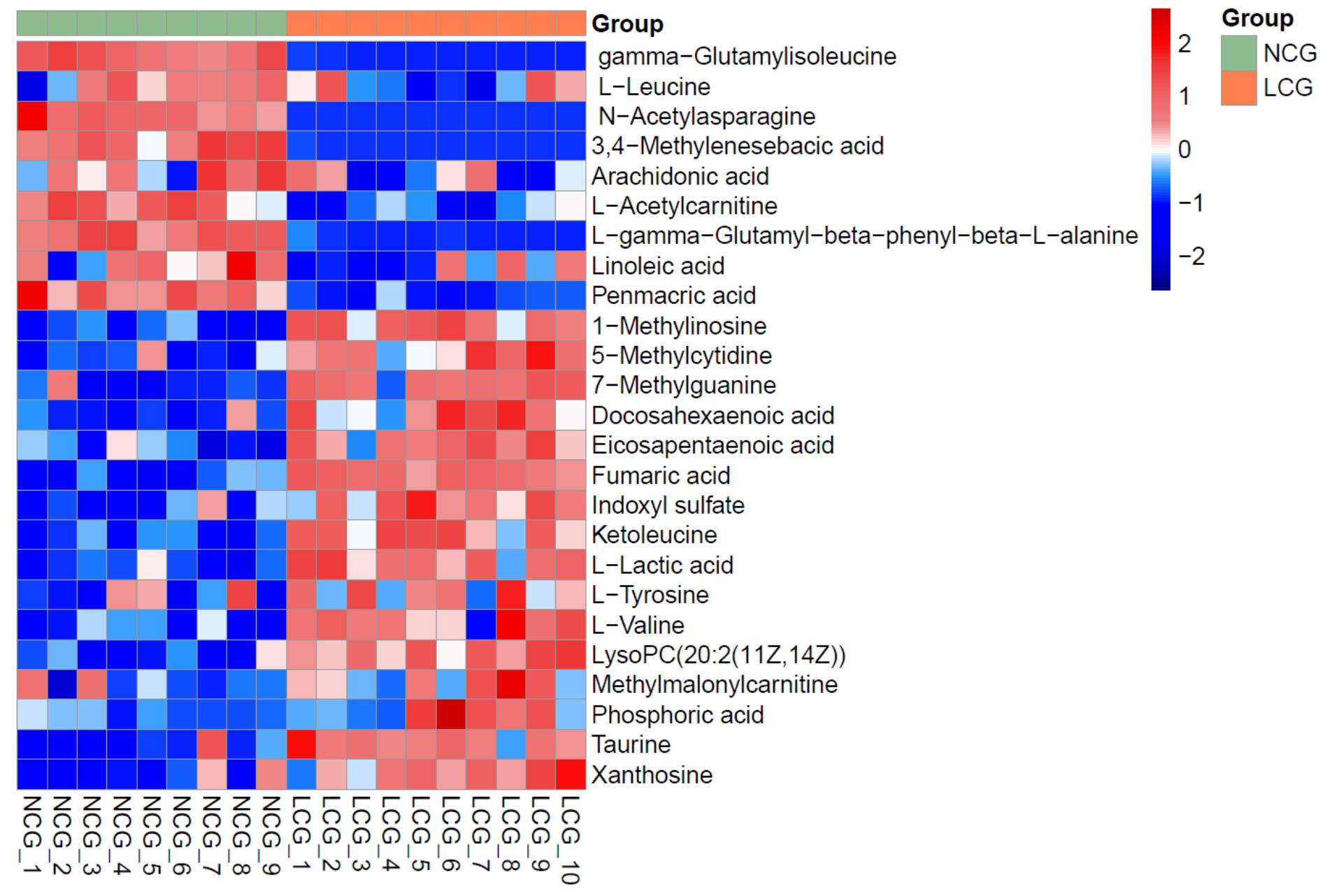

\section{Figure 3}

Heatmap of the identified 25 biomarkers of calcium deficiency in serum. Rows: biomarkers; columns: samples. Green: NCG; red: LCG. The color key indicates the metabolite expression value: dark blue, lowest; dark red, highest. 
A

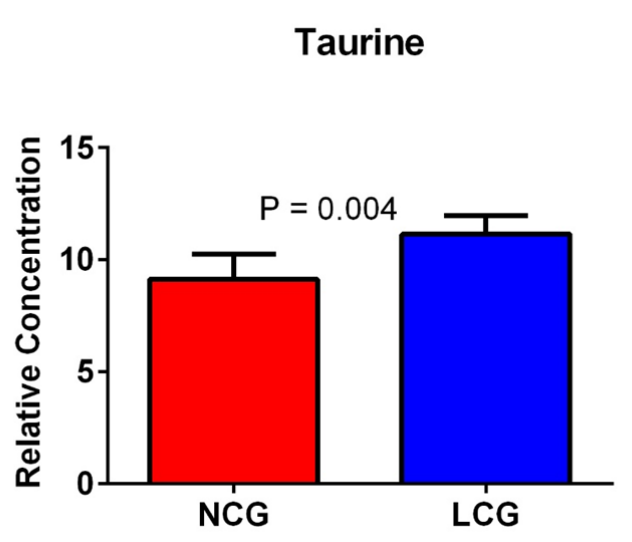

B

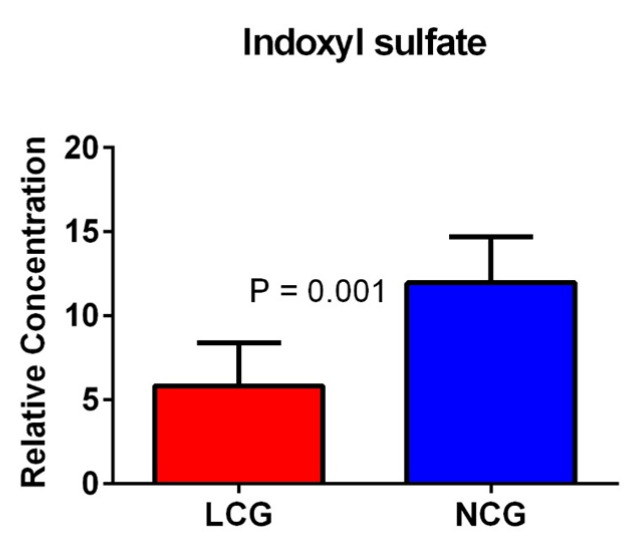

C

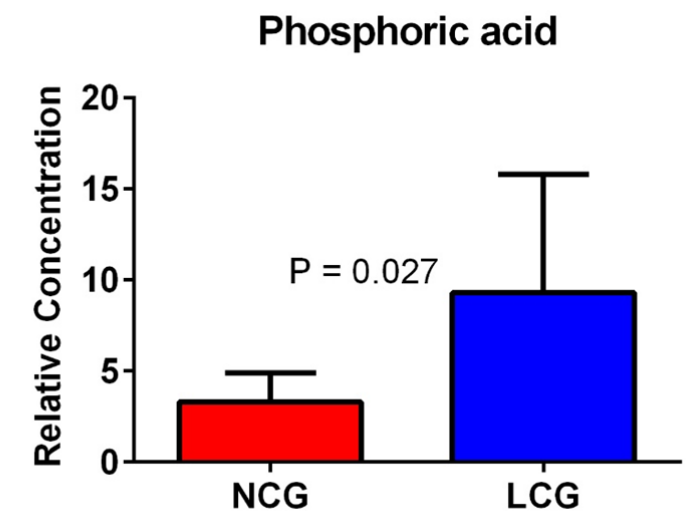

D
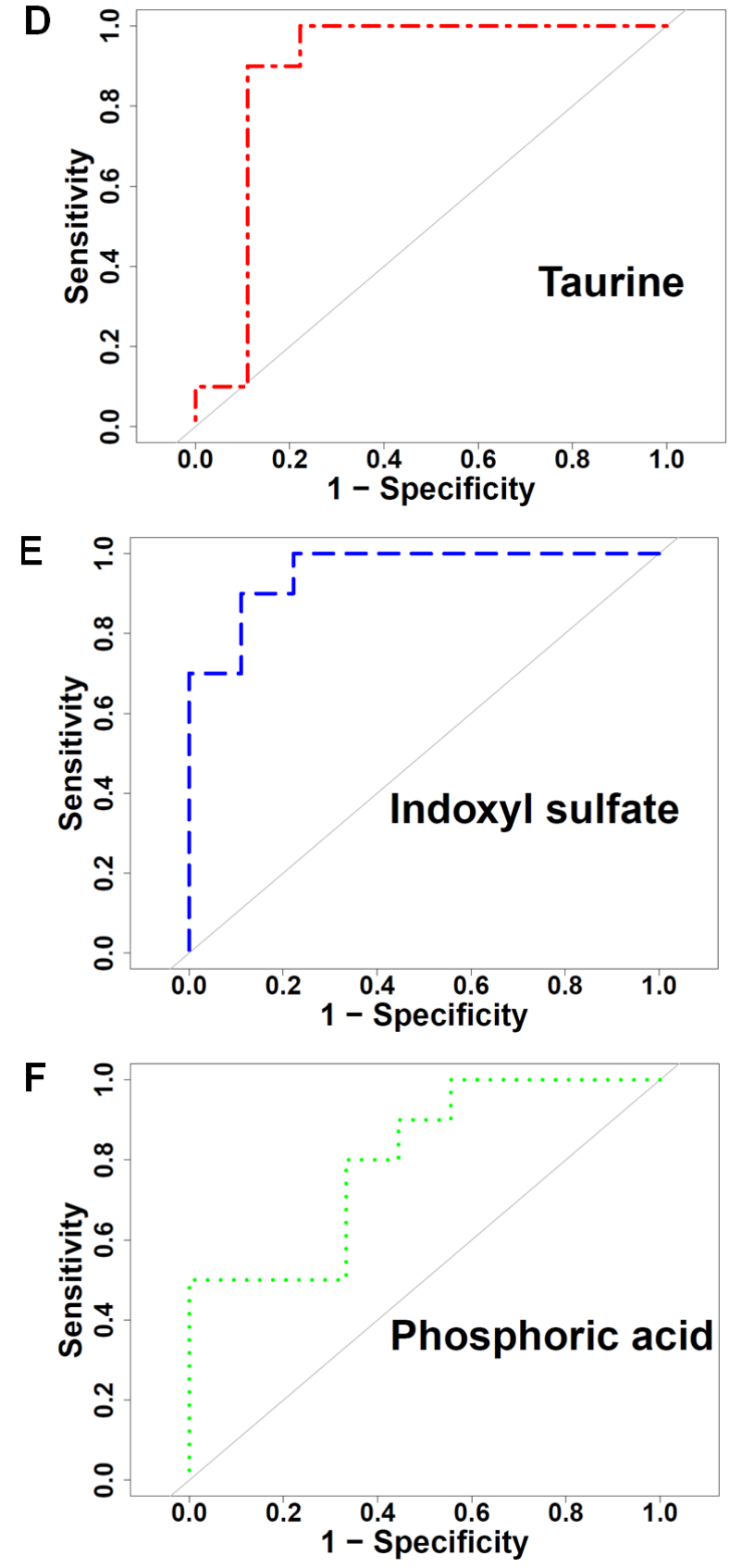

Figure 4

Areas under the ROC curves of three biomarkers. 


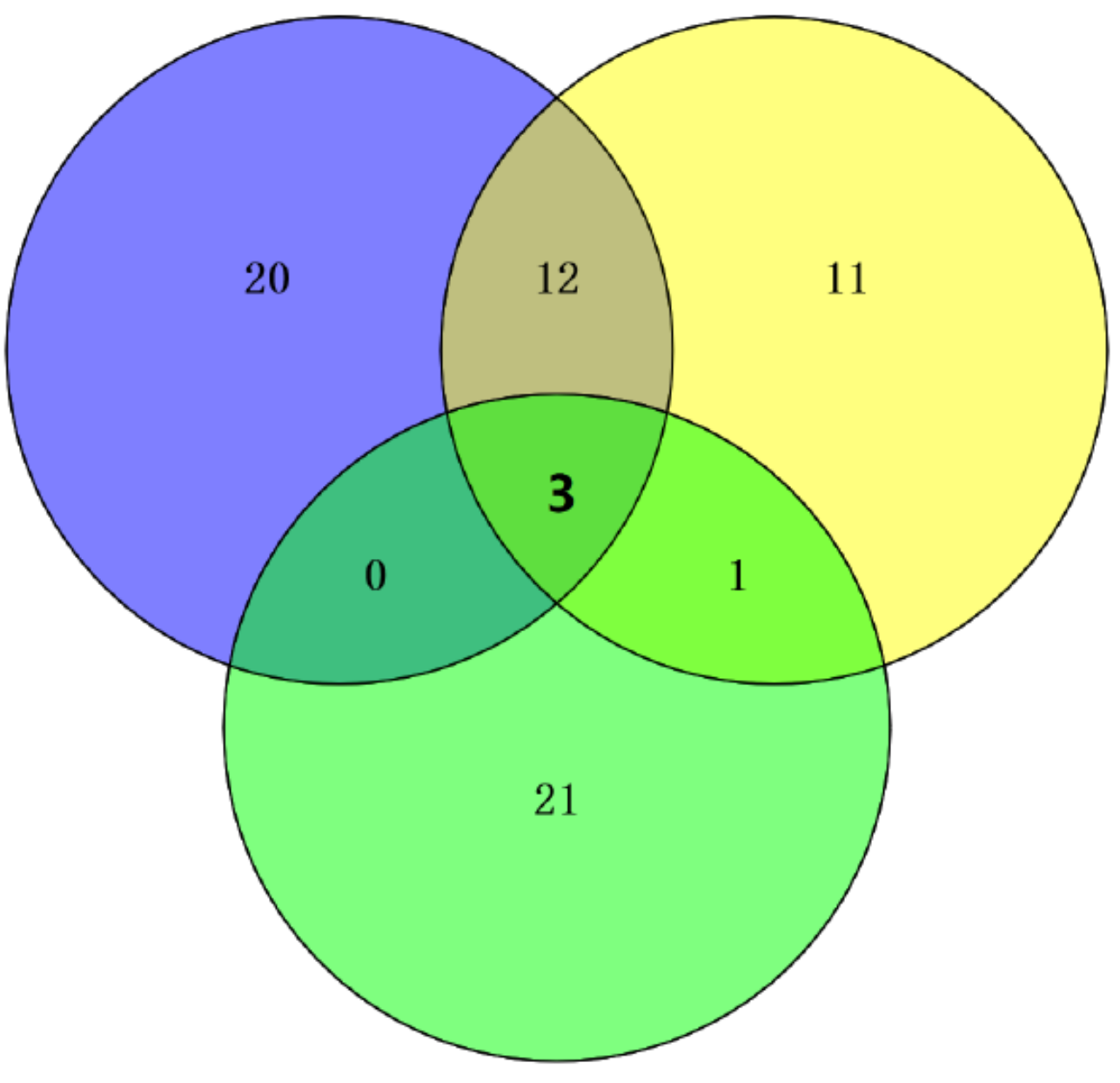

Figure 5

Venn diagram of the urine and blood biomarkers of the calcium-deficient rat and the urinary biomarkers of rickets. 


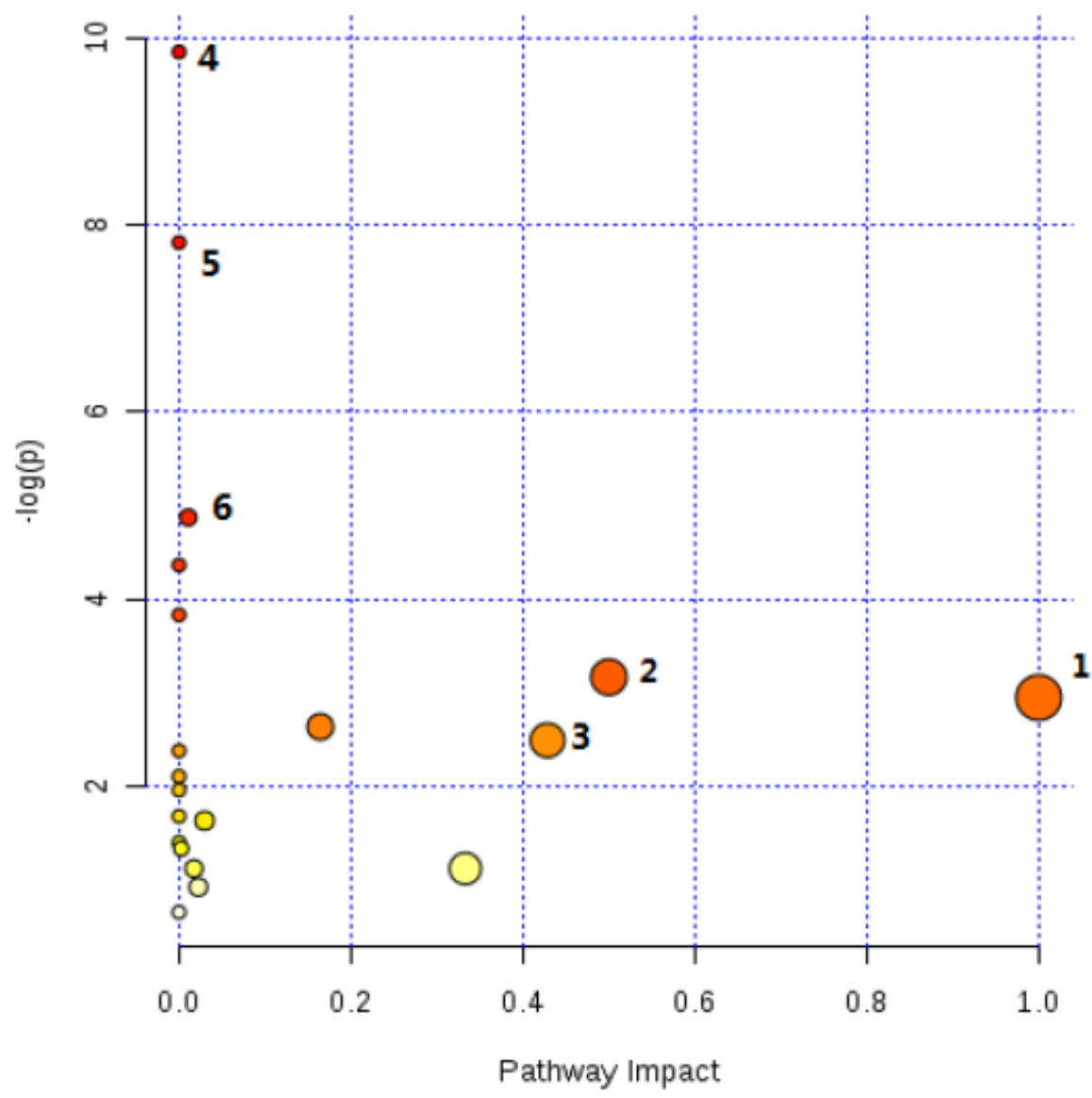

\section{Figure 6}

Summary of pathway analysis with MetaboAnalystR. 1.Linoleic acid metabolism; 2. Phenylalanine, tyrosine and tryptophan biosynthesis; 3 . Taurine and hypotaurine metabolism; 4. Valine, leucine and isoleucine biosynthesis; 5 . Biosynthesis of unsaturated fatty acids; 6 . Valine, leucine and isoleucine degradation.

\section{Supplementary Files}

This is a list of supplementary files associated with this preprint. Click to download.

- SupplementaryTable14.docx

- SupplementaryData1.xIsx 